\title{
An Alternative Method for Continuous Property Measurement during Depth- sensing Indentation
}

\author{
Y.L.Chiu ${ }^{1}$, G. Feng ${ }^{2}$, B. Tang ${ }^{3}$, A.H.W. Ngan ${ }^{3, *}$ \\ ${ }^{1}$ Department of Chemical Engineering and Materials, The University of Auckland, Private \\ Bag 92019, Auckland City, New Zealand \\ ${ }^{2}$ Engineering Division, Brown University, Providence, RI 02912, USA \\ ${ }^{3}$ Department of Mechanical Engineering, The University of Hong Kong, Pokfulam Road, \\ Hong Kong, P.R. China \\ * Contact Author (Email: hwngan@hku.hk)
}

\begin{abstract}
Based on the Oliver-Pharr framework, we propose an iteration scheme to continuously evaluate the hardness during monotonic loading in nanoindentation. The new method has been applied to measure the hardness of various crystalline materials including $\mathrm{Al}, \mathrm{Cu}, \mathrm{Ni}_{3} \mathrm{Al}(\mathrm{Cr}, \mathrm{B})$ and $\mathrm{MgO}$, where an obvious indentation size effect (ISE) has been characterized. The results on MgO measured using the new method show excellent agreement with those from the more conventional, dynamic Continuous Stiffness Measurement (CSM) method. In $a$-Se and PP, an apparent ISE was observed when constant-rate loading schedules were used. However, no ISE was observed in these two materials when using exponential loading schemes which corresponded to constant strain rates. The apparent ISE under constant-rate loading was thus very likely the result of the strong strain-rate dependence of the flow resistance in these two materials. The new method therefore produced the correct ISE behaviours in various materials under different loading situations.
\end{abstract}

Keywords: Nanoindentation, hardness, indentation size effect, metals, polymers 


\section{Authors' brief biographical notes:}

Y.L. Chiu, BEng, PhD, is a Lecturer at the Department of Chemical and Materials Engineering, University of Auckland. His main research interest includes electron microscopy of materials, microstructure-property relations and nanoindentation. G. Feng, BEng, MPhil, PhD, is a postdoctoral researcher at the Engineering Division, Brown University. His interest includes nanoindentation and related micromechanics issues. B. Tang, $\mathrm{BEng}, \mathrm{PhD}$, is a postdoctoral researcher at the Department of Mechanical Engineering, the University of Hong Kong. His interest includes the development of nanoindentation techniques in soft materials, and biomaterials in general. A.H.W. Ngan, BSc(Eng), PhD, MIMMM, CSci, is Professor at the Department of Mechanical Engineering, University of Hong Kong. His research interest includes

electron microscopy of materials, dislocation theory, microstructure-mechanical property relationship, nanoindentation, and so on. 


\section{Introduction}

Hardness has been one convenient parameter to represent the strength of materials. Hardness is conventionally defined as the mean pressure when a load is applied onto a specimen surface through a small probe, i.e. $H=P$ / $A$, where $P$ is the applied load and $A$ the indent area. In depth-sensing nanoindentation, which is more widely known as nanoindentation, $A$ is replaced by the tip-sample contact area, $A_{c}$, at full load (Doerner and Nix 1986; Oliver and Pharr 1992). Depth-sensing nanoindentation has been widely used for the measurement of mechanical properties from very small volumes of materials. In this technique, the applied load $P$ and the indenter displacement $h$ are logged continuously with respect to time $t$. The standard method to analyze the $P(t)$ and $h(t)$ data to yield material properties is the one proposed by Oliver and Pharr (Oliver and Pharr 1992). In this method, the tip-sample contact stiffness $S$ is evaluated from the onset of unloading from the peak load, and then the contact area $A_{c}$ is evaluated from $S$.

A major limitation of the Oliver-Pharr method is that material properties can only be evaluated at the peak load. In materials research, however, it is often necessary to characterize the properties continuously along the loading path, for example, to investigate whether material properties would vary as a function of the deformation volume size, i.e. the so-called indentation size effect (ISE). One usual method this can be done is the continuous stiffness measurement (CSM) method (Oliver and Pethica), in which the tip-sample contact stiffness is measured continuously through the use of a small-amplitude, high-frequency sinusoidal signal superimposed onto the main loading schedule. Provided that the damping coefficient and compliance of the machine are accurately calibrated beforehand, the dynamic response of the tip can be used to work out the contact stiffness continuously.

The dynamic CSM module, however, is usually not a standard feature in commercial nanoindenters, but is a rather expensive, add-on option. The need to carefully calibrate the damping and compliant characteristics should also not be overemphasized. In this paper, we present an alternative, cheaper way of carrying out continuous property measurement using a standard, static testing module. This method is based on simple manipulation of the contactmechanics equations involved in the traditional Oliver-Pharr (O-P) method, and therefore should have the accuracy equivalent to that of the O-P method. Since the O-P method is still the 
"golden" standard in nanoindentation analysis, we believe that the method proposed here is based on very solid grounds.

In the next section, we first derive the main equation in the new method. Then, we apply the new method to a range of materials with known indentation size effect (ISE) behaviours.

\section{The New Method}

The Sneddon solution to the elastic contact problem between an axisymmetric tip and a flat surface (Sneddon 1965) gives the following expression for the reduced modulus of contact:

$$
E_{r}=\frac{\sqrt{\pi}}{2} \frac{S}{\sqrt{A_{c}}},
$$

where $S$ is the contact stiffness and $A_{c}$ the contact area under load. In nanoindentation, the contact area is obtained via a pre-calibrated shape function of the tip, such as

$$
A_{c}=A\left(h_{c}\right)=C_{0} h_{c}^{2}+C_{1} h_{c}+C_{2} h_{c}^{1 / 2}+C_{3} h_{c}^{1 / 4}+C_{4} h_{c}^{1 / 8}+C_{5} h_{c}^{1 / 16}+\cdots,
$$

where $C_{n}$ are fitting constants, and $h_{c}$ is the contact depth. Oliver and Pharr (Oliver and Pharr 1992) derived an approximate expression of $h_{c}$ as

$$
h_{c}=h_{\max }-\varepsilon \frac{P_{\max }}{S}
$$

where $P_{\max }$ and $h_{\max }$ are the maximum load and the corresponding tip displacement respectively, and $\varepsilon=0.75$ for a Berkovich indenter.

In the O-P method, the contact stiffness $S$ is evaluated by unloading from $\left(P_{\max }, h_{\max }\right)$, and in deriving eqn. (3), Oliver and Pharr made certain assumptions regarding the unloading curve from $\left(P_{\max }, h_{\max }\right)$. But the final result in the form of eqn. (3) does not contain parameters 
pertinent to the unloading, apart from $S$, but then it is the instantaneous contact stiffness at ( $P_{\max }$, $\left.h_{\max }\right)$. We therefore note that eqns. (1) and (3) are actually valid for each pair of $(P, h)$ data during a monotonically increasing load schedule, i.e. not just at $\left(P_{\max }, h_{\max }\right)$, and these two

equations in fact are the basis of the dynamic CSM method. Thus, replacing $\left(P_{\max }, h_{\max }\right)$ by $(P, h)$ in eqn. (3), and combining with eqn. (1), give

$$
h_{c}=h-\varepsilon \frac{P \sqrt{\pi}}{2 E_{r} \sqrt{A\left(h_{c}\right)}} .
$$

If $E_{r}$ is known, eqn. (4), together with eqn. (2), provide an iterative scheme by which the contact depth $h_{c}$ can be solved for each pair of $(P, h)$ data along a monotonic increasing load schedule. Once $h_{c}$ is known, $A_{c}$ can be computed from eqn. (2), and then hardness can be evaluated continuously as $H=P / A_{c}$, as usual. For an unknown material, the $E_{r}$ can be obtained using the usual O-P method from the final unloading from the peak load.

The new method here is therefore very simple and can be easily incorporated into the analysis software of any nanoindenter. As mentioned above, only equations fundamental to the O-P method are involved, and so the method here suffers from the same uncertainties as the latter, for example, the inability to account for pile-up type of indents, effects of thermal drifts on the $h$ data, and so on. Furthermore, when using eqn. (4) as an iterative scheme to find $h_{c}$, it is assumed that $E_{r}$ does not vary with the indentation depth, i.e. the method is applicable to homogeneous materials, but not necessarily to heterogeneous materials in which the elastic properties may change as a function of depth into the surface.

\section{Materials and Experiments}

To illustrate the applicability of the new method, nanoindentation experiments were carried out in a number of samples, including:

(i) a high-purity (99.99\%) (111) oriented single crystal copper,

(ii) a (111) oriented single crystal of $\mathrm{Ni}_{3} \mathrm{Al}(\mathrm{Cr}, \mathrm{B})$,

(iii) a polycrystalline aluminum sample of commercial purity,

(iv) a (001) oriented MgO single crystal, 
(v) an amorphous selenium sample, and,

(vi) a polypropylene (PP) sample.

The reason for choosing such a range of materials is because these materials are known to exhibit very different ISE behaviours. For the crystalline samples (i) to (iv) above, it is by now well-known that the nanoindentation hardness decreases as the indentation depth increases (Nix and Gao 1998; Kim et al. 2005; Lu et al. 2006; Manika and Maniks 2006; Scholz et al. 2006). For instance, based on the work-hardening effects of geometrically necessary dislocations, Nix and Gao (Nix and Gao 1998) proposed that the ISE of hardness in these materials obeys $H=H_{o} \sqrt{1+h^{*} / h}$, where $H_{o}$ is the hardness in the limit of infinite depth, and $h^{*}$ is a characteristic length scale. The Nix-Gao equation was found to be valid for indents deeper than about $1 \mu \mathrm{m}$, but for indents shallower than this, it usually overestimates the hardness (Swadener et al. 2002). For amorphous or semi-amorphous materials such as (v) or (vi) above, no strong ISE should be expected. The purpose of studying these materials was therefore to see whether the new method can successfully reproduce the different expected types of ISE in different materials. Comparison with the dynamic CSM method was also made to check for any discrepancies.

Sample preparation procedures have already been described in detail elsewhere (Feng and Ngan 2001; Liu and Ngan 2001; Feng and Nix 2004) and are not repeated here. Depthsensing nanoindentation tests were carried out on either a Hysitron Triboscope transducer mounted on an atomic-force microscope supplied by Park Scientific Instruments, a Nanohardness Tester supplied by CSEM Instrument SA Co., or an MTS Nanoindenter XP equipped with a dynamic CSM module. Berkovich diamond indenters were used on all three machines, and the tip-area functions $A_{c}\left(h_{c}\right)$ were calibrated using the standard procedure (Oliver and Pharr 1992). Thermal drift was either corrected by a low-load holding stage, e.g. at $10 \%$ of the maximum load, during the unloading process, or was simply ignored when the entire test was within a short duration such as 5 seconds.

For viscoelastic materials such as Se or PP, there is a well-known subtlety concerning whether an ISE on hardness is to be observed or not. In these materials, the flow resistance decreases as the strain rate slows down, and so an artificial change in hardness can be expected 
to be observed if the strain rate is not maintained constant. This change is not due to an internal length scale effect as in the Nix-Gao model (Nix and Gao 1998), and hence we do not describe it as an intrinsic ISE. The strain-rate field during indentation by a self-similar indenter is known to scale with $(1 / h)(d h / d t)$ or $(1 / P)(d P / d t)$ (Mayo and Nix 1988; Lucas and Oliver 1999). Hence, if a constant loading rate $d P / d t$ is used, for example, the strain rate will drop as loading proceeds. To keep the strain rate constant, one will have to use an exponential load function $P \sim \exp (k t)$, where the constant $k=(1 / P)(d P / d t)$ is now a measure of the (constant) strain rate. In the present experiments, both constant- and exponential-loading-rate schemes were used for the Se and PP samples to see if the proposed analysis method could indeed give the correct ISE.

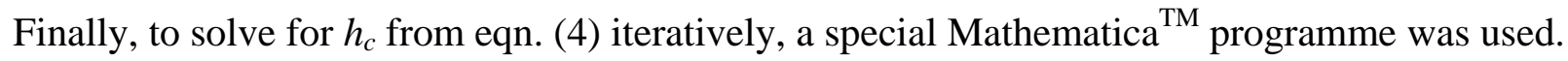

\section{Results and discussion}

Fig. 1(a-c) show the hardness versus depth $(H-h)$ curves during constant-rate loading for the three metals obtained using the present proposed method. The elastic moduli obtained using the O-P method from the final unloading are $201.86 \mathrm{GPa}$ for (111) $\mathrm{Ni}_{3} \mathrm{Al}(\mathrm{Cr}, \mathrm{B}), 125.87 \mathrm{GPa}$ for (111) $\mathrm{Cu}$, and $75 \mathrm{GPa}$ for $\mathrm{Al}$ respectively. These values are in good agreement with literature values (Feng and Ngan 2002), giving one the confidence of using the O-P method for these materials. The data in Fig. 1(a-c) clearly indicate that very strong ISE exists for the three metals studied.

To see whether the new method can produce results compatible with the dynamic CSM method, constant-strain-rate experiments were carried out on the Nanoindenter XP using the CSM mode. Fig. 2 shows the ISE measured from the MgO single crystal at a constant strain rate of $k=(1 / P)(d P / d t)=0.05 \mathrm{~s}^{-1}$. The black solid line shows the hardness data calculated using the new method, and the grey square symbols shows the hardness data computed using the conventional, dynamic CSM method. It can be seen that the hardness data calculated using the two methods are in excellent agreement.

In the amorphous Se ( $a$-Se) sample, no intrinsic ISE should be expected but an apparent ISE can be produced by using a constant-rate loading schedule, as shown in fig. 3(a). Here, the loading rate was constant and so the strain rate should drop as $1 / P$. The strong reduction in the 
hardness measured using the new method should therefore be due to the strain-rate effect on the flow resistance in $a$-Se, which is supposed to undergo viscous deformation at room temperature (Poisl et al. 1995; Roland et al. 1999). However, when an exponential loading schedule was used, the measured hardness became constant with respect to load, as shown in fig. 3(b). Here, $k=(1 / P)(d P / d t)=0.08 \mathrm{~s}^{-1}$, and it can be seen that the hardness stays constant at about $0.4 \mathrm{GPa}$ during the exponential loading. The fact that the new method here can reproduce the expected behaviours in the two loading situations illustrates the validity of the method.

More similar experiments were carried out in another (partially) amorphous material PP, in which no intrinsic ISE should exist. Fig. 4(a) shows the apparent ISE observed when constantrate loading schedules were used. Here, the loading rate ranged from 10 to $1600 \mathrm{mN} / \mathrm{min}$. It can be seen that the higher the loading rate used, the more obvious is the apparent ISE. This is reasonable since the change in the strain rate within the same load range scales with the loading rate. Similar to that of $a$-Se, when exponential loading schemes were used, the hardness stayed nearly constant, as shown in fig. 4(b). Here, three values of $k=(1 / P)(d P / d t)$ were used, and it can be seen that the flow resistance of PP exhibits a strong dependence on $k$ or the strain rate. Again, we see that the new method here produced the expected results in PP at different loading situations.

The loading curves of nanoindentation tests have been analyzed by other researchers in order to derive the mechanical properties of interest. Hainsworth et al. (Hainsworth et al. 1996) found that the loading curve could be described as $P=K_{m} h^{2}$, where $K_{m}$ is a function of the elastic modulus and hardness. Therefore, knowing either the elastic modulus or the hardness, the other one could be derived from the loading curve. However, as the authors admitted, the assumption of a constant hardness regardless of the loading scale is not always right. Based on dimensional analysis and finite element analysis, Cheng and Cheng (Cheng and Cheng 1998; Cheng and Cheng 1998; Cheng and Cheng 1999) provided an alternative method to derive mechanical properties from a nanoindentation loading curve. The loading curve is characterized in the form $P=E h^{2} \Pi(Y / E, v, \theta)$, where the yield strength $Y$, elastic modulus $E$ and the Poisson ratio $v$ can be related together with $\theta$, the indenter characteristic angle. Nevertheless, unique determination of $E, Y, v, \theta$ from a single loading curve is difficult. Compared with these 
previous methods which often require more complex analyses and calculations, the iteration scheme proposed in the present work needs minimum work since the hardness can be extracted from the loading curve without knowing any empirical constitutive law for the deformation.

\section{Conclusions}

The following points can be concluded from the present work:

1) An iteration scheme based on the Oliver-Pharr framework has been proposed to continuously evaluate the hardness during monotonic loading in nanoindentation.

2) The hardness data measured using the new method are in excellent agreement with the usual, dynamic CSM method.

3) The hardness results obtained using the proposed scheme from $\mathrm{Al}, \mathrm{Cu}, \mathrm{Ni}_{3} \mathrm{Al}(\mathrm{Cr}, \mathrm{B})$ and $\mathrm{MgO}$ all show an obvious ISE, as expected.

4) In $a$-Se and PP, an apparent ISE was observed when constant-rate loading schedules were used. This is presumably a manifestation of a strong strain-rate effect on the flow resistance in these two materials.

5) In $a$-Se and PP, no ISE was observed when using exponential loading schedules which corresponded to constant strain rates. The new method therefore produced the correct ISE behaviours in the two loading situations.

\section{Acknowledgments}

The work described in this paper was partially supported by a grant from the Research Grants Council of the Hong Kong Special Administrative Region, China (Project no. HKU7167/05E) and an ISAT grant from the Royal Society of New Zealand (Project no. 9071/3606251). The indentation data for MgO were obtained using the facilities at Stanford University during G. Feng’s PhD study under the guidance of Prof. W. D. Nix.

\section{References}


Cheng, Y.-T. and C.-M. Cheng (1998). "Analysis of indentation loading curves obtained using conical indenters." Philosophical Magazine Letters 77(1): 39-47.

Cheng, Y.-T. and C.-M. Cheng (1998). "Further analysis of indentation loading curves: Effects of tip rounding on mechanical property measurements." Journal of Materials Research 13(4): 1059-1064.

Cheng, Y.-T. and C.-M. Cheng (1999). "Can stress-strain relationships be obtained from indentation curves using conical and pyramidal indenters?" Journal of Materials Research 14(9): 3493-3496.

Doerner, M. F. and W. D. Nix (1986). "A method for interpreting the data from depth-sensing indentation instruments." Journal of Materials Research 1(4): 601.

Feng, G. and A. H. W. Ngan (2001). "Creep and strain burst in indium and aluminium during nanoindentation." Scripta Materialia 45(8): 971-976.

Feng, G. and A. H. W. Ngan (2002). "Effects of creep and thermal drift on modulus measurement using depth-sensing indentation." Journal of Materials Research 17(3): 660-668.

Feng, G. and W. D. Nix (2004). "Indentation size effect in MgO." Scripta Materialia 51(6): 599603.

Hainsworth, S. V., H. W. Chandler, et al. (1996). "Analysis of nanoindentation loaddisplacement loading curves." Journal of Materials Research 11(8): 1987-1995.

Kim, J.-Y., D. T. Read, et al. (2005). Depth-dependent hardness characterization by nanoindentation using a berkovich indenter with a rounded tip. Materials Research Society Symposium Proceedings.

Liu, Y. and A. H. W. Ngan (2001). "Depth dependence of hardness in copper single crystals measured by nanoindentation." Scripta Materialia 44(2): 237-241.

Lu, C., Y.-W. Mai, et al. (2006). "Indentation size effect on hardness of nanostructured thin films." Key Engineering Materials 312: 363-368. 
Lucas, B. N. and W. C. Oliver (1999). "Indentation power-law creep of high-purity indium." Metallurgical and Materials Transactions a-Physical Metallurgy and Materials Science 30(3): 601-610.

Manika, I. and J. Maniks (2006). "Size effects in micro- and nanoscale indentation." $\underline{\text { Acta }}$ Materialia 54(8): 2049-2056.

Mayo, M. J. and W. D. Nix (1988). "A micro-indentation study of superplasticity in Pb, Sn, and Sn-38 wt\% Pb." Acta Metallurgica 36(8): 2183-2192.

Nix, W. D. and H. Gao (1998). "Indentation size effects in crystalline materials: A law for strain gradient plasticity." Journal of the Mechanics and Physics of Solids 46(3): 411-425.

Oliver, W. C. and J. B. Pethica (1989). "Method for continuous determination of the elastic stiffness of contact between two bodies." US Patent No. 4848141.

Oliver, W. C. and G. M. Pharr (1992). "An Improved Technique for Determining Hardness and Elastic-Modulus Using Load and Displacement Sensing Indentation Experiments." Journal of Materials Research 7(6): 1564-1583.

Poisl, W. H., W. C. Oliver, et al. (1995). "Relationship between indentation and uniaxial creep in amorphous selenium." Journal of Materials Research 10(8): 2024-2032.

Roland, C. M., P. G. Santangelo, et al. (1999). "Creep of selenium near the glass temperature." Journal of Chemical Physics 111(20): 9337-9342.

Scholz, T., J. Munnoz-Saldanna, et al. (2006). "Indentation size effect in barium titanate with spherical tipped nanoindenters." Applied Physics Letters 88(9).

Sneddon, I. N. (1965). "The relation between load and penetration in the axisymmetric Boussinesq problem for a punch of arbitrary profile." Int. J. Eng. Sci. 3: 47-57.

Swadener, J. G., E. P. George, et al. (2002). "The correlation of the indentation size effect measured with indenters of various shapes." Journal of the Mechanics and Physics of Solids 50(4): 681-694. 


\section{Figure Captions:}

Fig. 1. The ISE observed in (a) single crystal copper, (b) polycrystalline aluminium and (c) single crystal $\mathrm{Ni}_{3} \mathrm{Al}(\mathrm{Cr}, \mathrm{B})$.

Fig. 2. The ISE observed in single crystal MgO tested at constant strain rate. Square grey symbols are data derived using continuous stiffness measurement (CSM). Black symbols are data obtained using the present iteration scheme (eqn.(4).

Fig. 3. (a) An apparent ISE observed in selenium with a constant-rate loading scheme. (b) No ISE is observed with a constant strain rate by setting $k=\dot{P} / P=0.08 \mathrm{~s}^{-1}$.

Fig. 4 (a) An apparent ISE in PP with constant-rate loading schemes. The ISE is more obvious at high loading rate. (b) Fairly constant hardness values measured at different indentation depth (i.e. no ISE) at constant strain rate by setting $k=\dot{P} / P=0.08 \mathrm{~s}^{-1}$. 

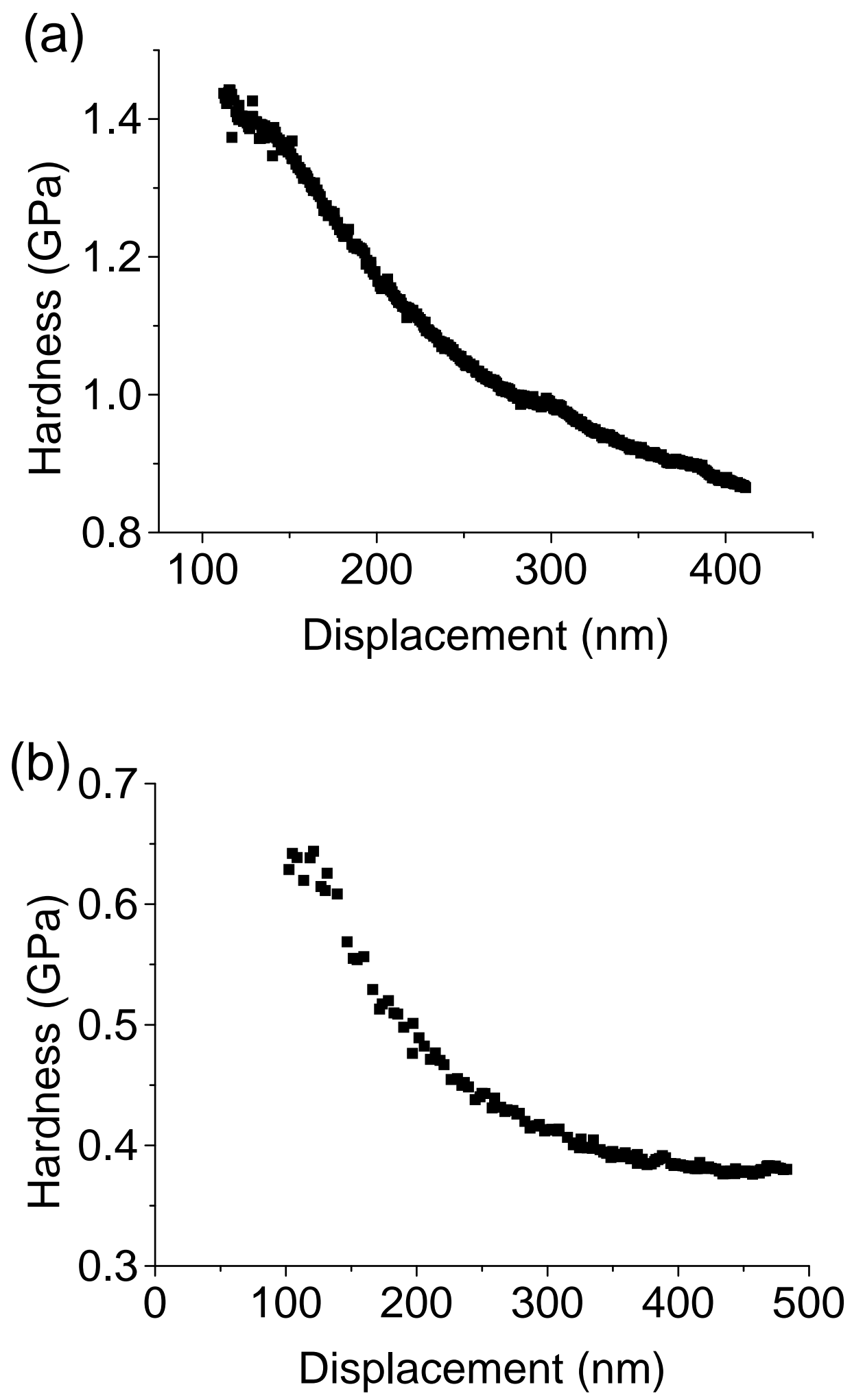

Figure 1

(Chiu, Feng, Tang and Ngan) 


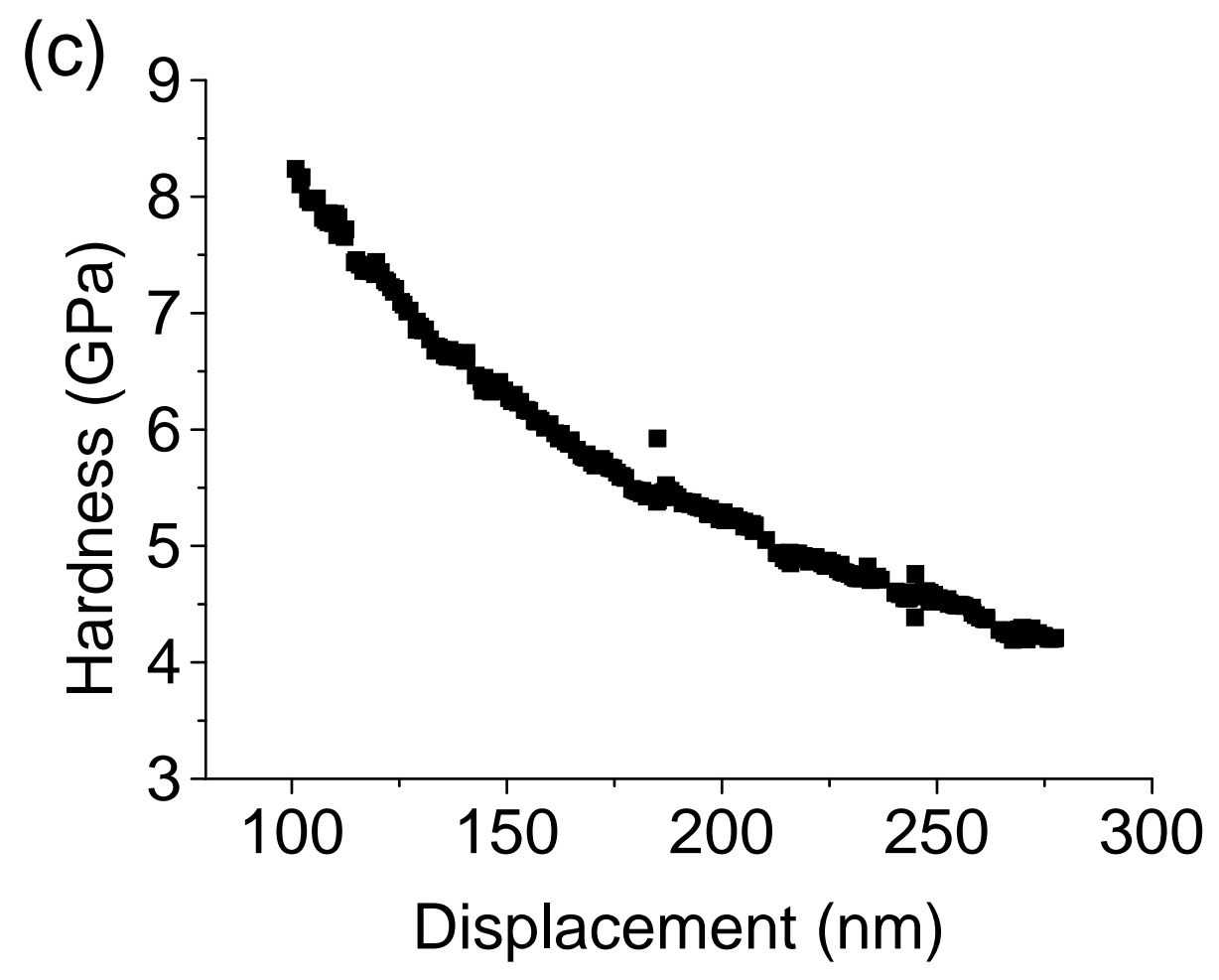

Figure 1

(Chiu, Feng, Tang and Ngan) 


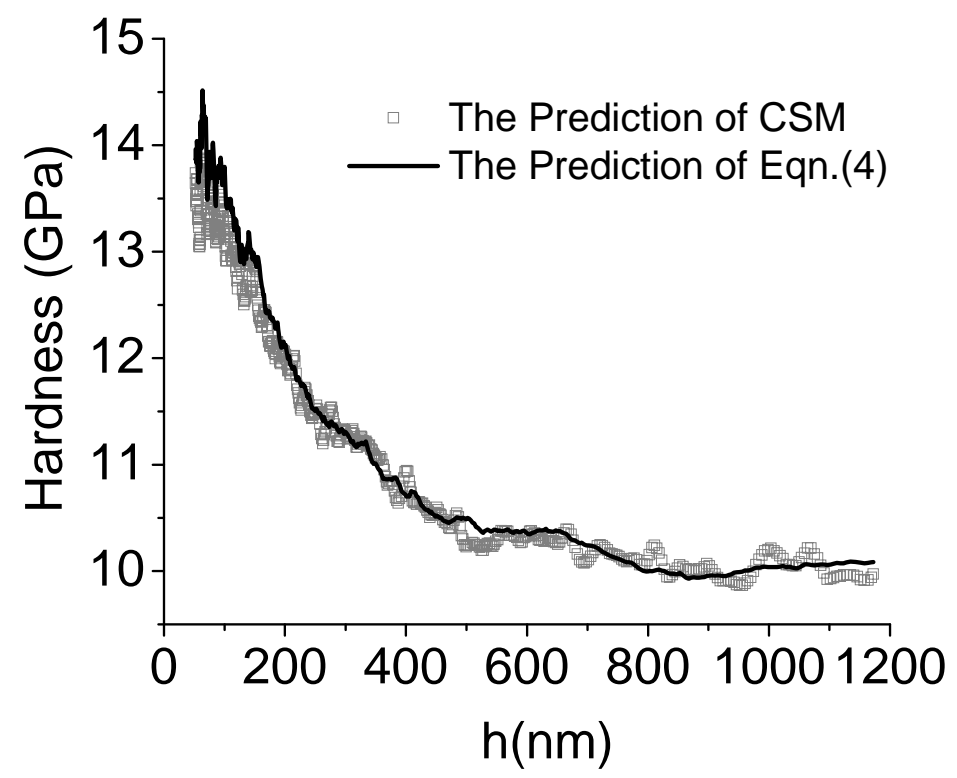

Figure 2

(Chiu, Feng, Tang and Ngan) 

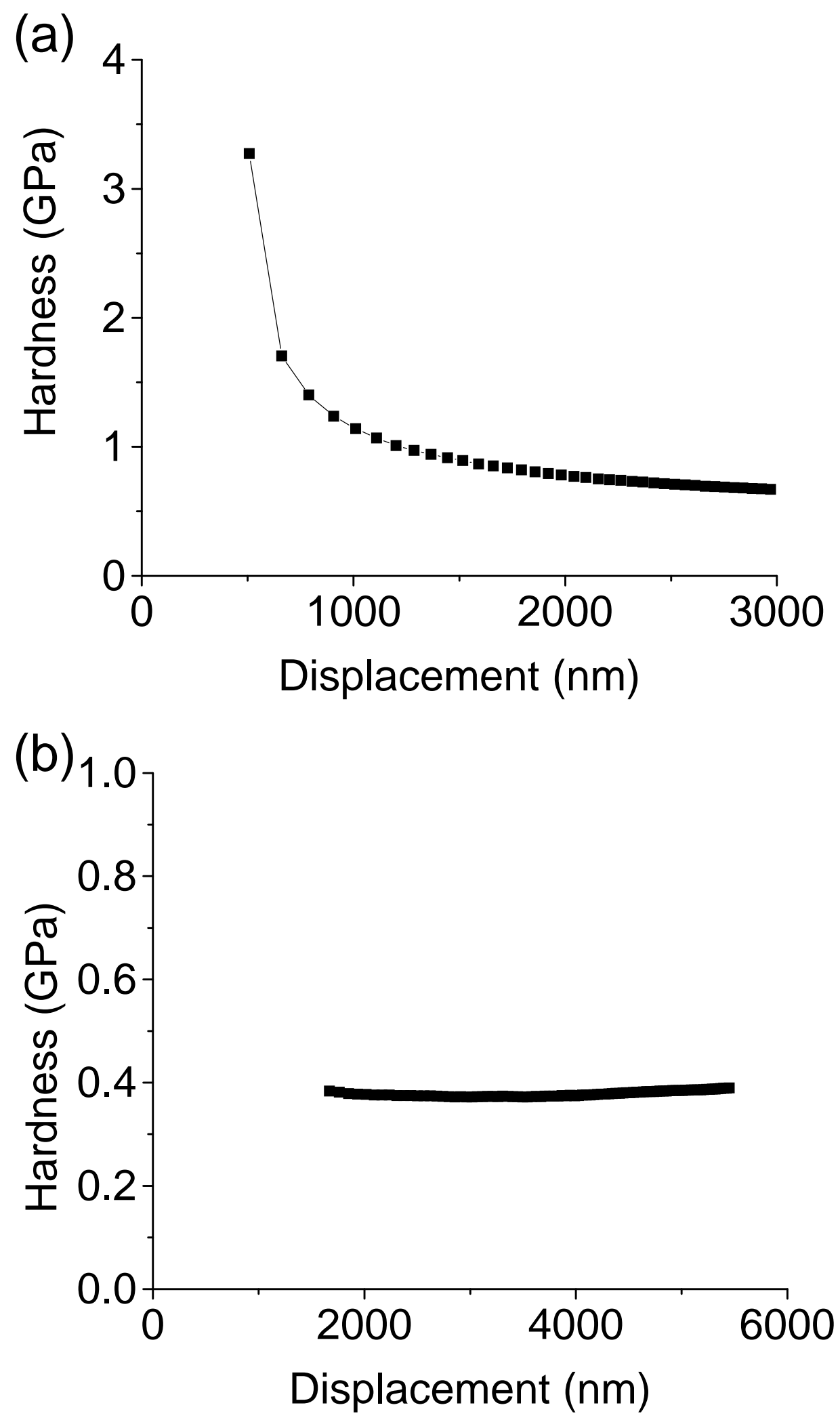

Figure 3

(Chiu, Feng, Tang and Ngan) 


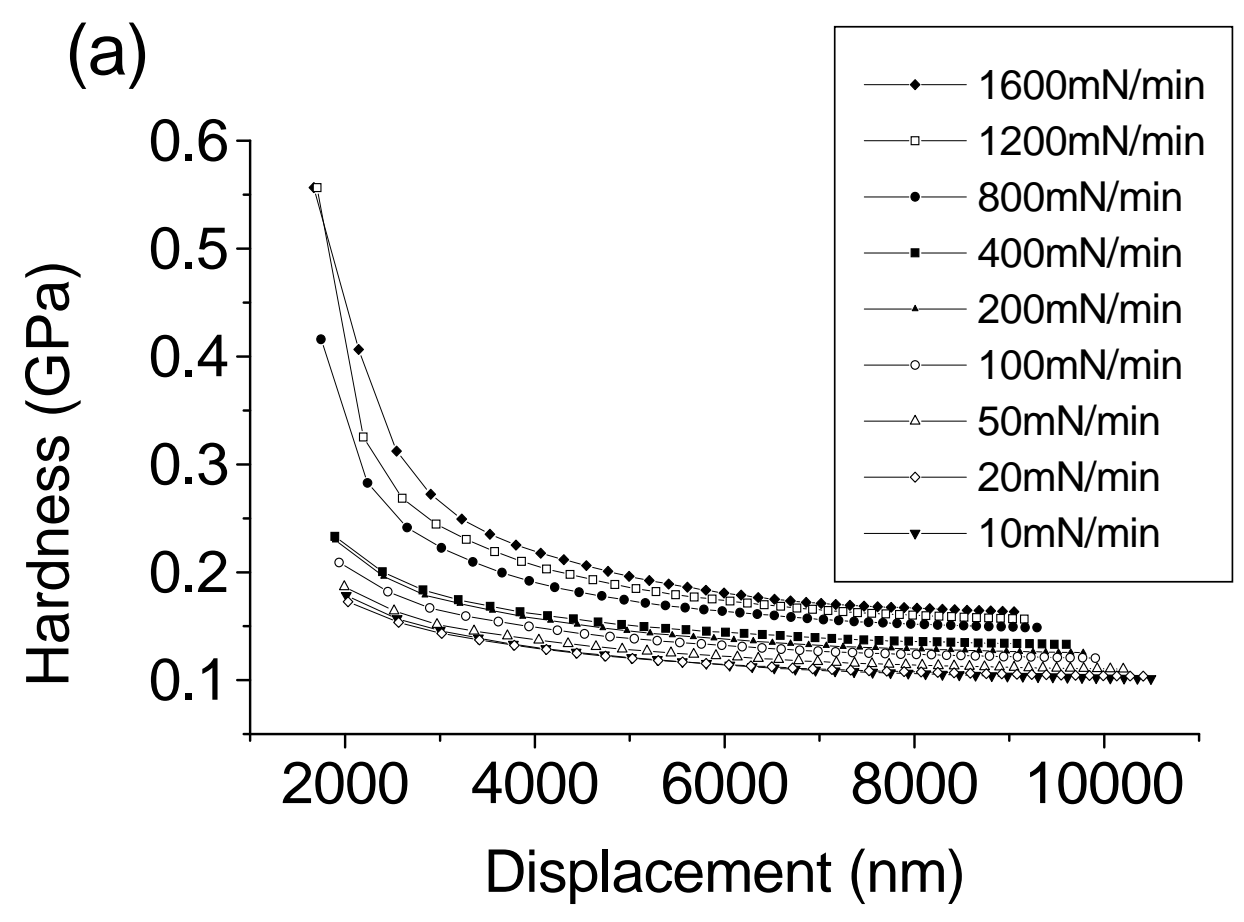

(b)

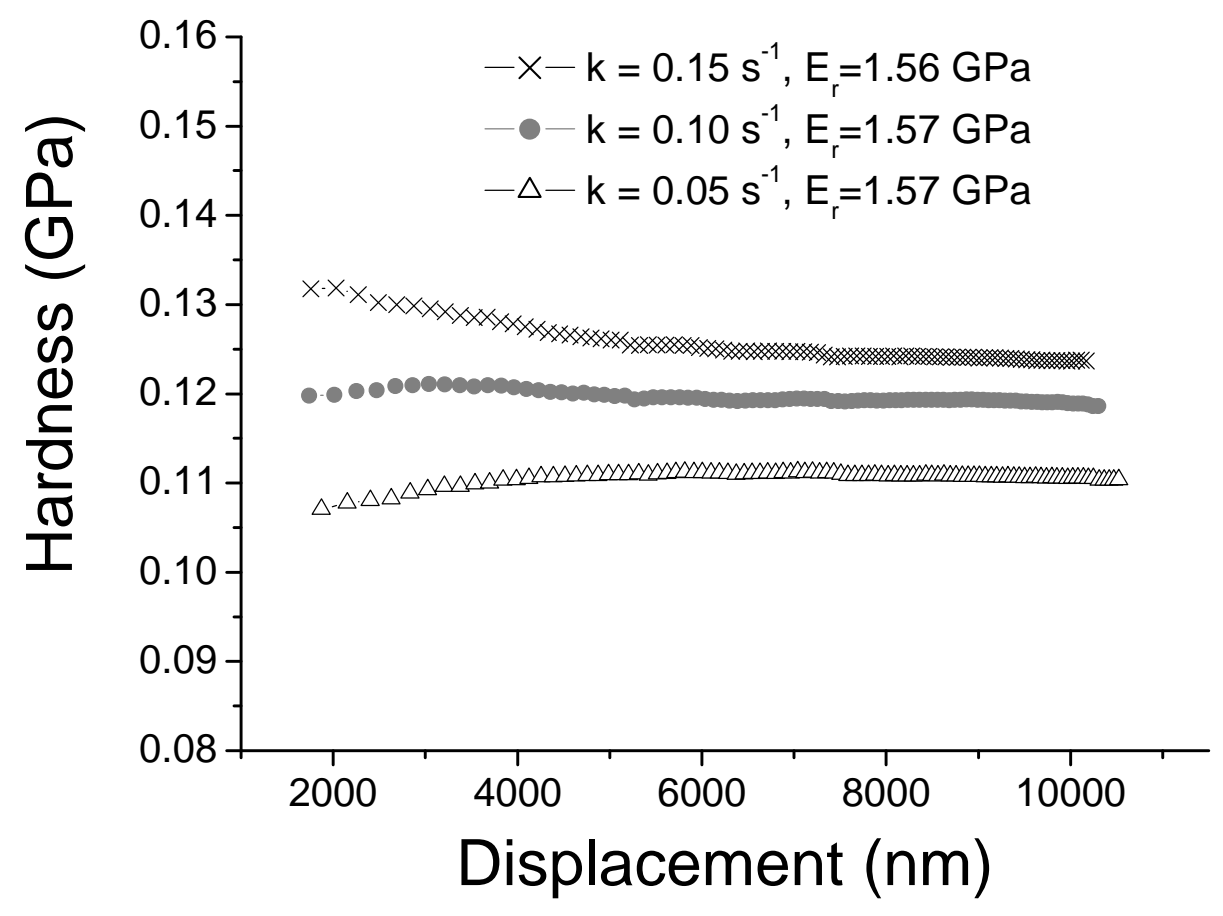

Figure 4

(Chiu, Feng, Tang and Ngan) 